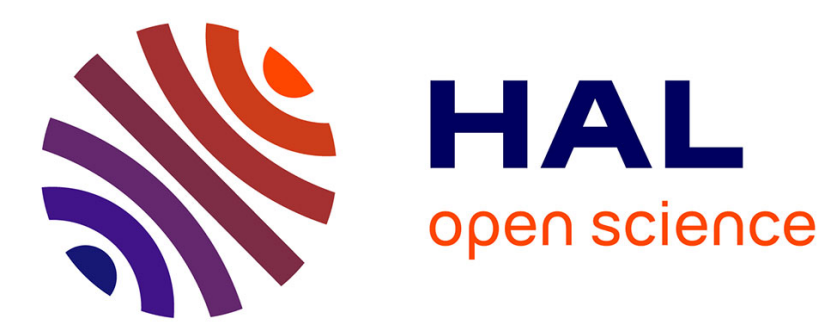

\title{
Some remarks on the stability of cell-to-cell spread models with distributed delays
}

Irinel-Constantin Morarescu, Silviu-Iulian Niculescu

\section{To cite this version:}

Irinel-Constantin Morarescu, Silviu-Iulian Niculescu. Some remarks on the stability of cell-to-cell spread models with distributed delays. 3rd IFAC Symposium on System Structure and Control, Oct 2007, Iguaçu, Brazil. 10.3182/20071017-3-BR-2923.00100 . hal-02293051

\section{HAL Id: hal-02293051 \\ https://hal.science/hal-02293051}

Submitted on 20 Sep 2019

HAL is a multi-disciplinary open access archive for the deposit and dissemination of scientific research documents, whether they are published or not. The documents may come from teaching and research institutions in France or abroad, or from public or private research centers.
L'archive ouverte pluridisciplinaire HAL, est destinée au dépôt et à la diffusion de documents scientifiques de niveau recherche, publiés ou non, émanant des établissements d'enseignement et de recherche français ou étrangers, des laboratoires publics ou privés. 


\title{
SOME REMARKS ON THE STABILITY OF \\ CELL-TO-CELL SPREAD MODELS WITH DISTRIBUTED DELAYS
}

\author{
Constantin-Irinel Morărescu, ${ }^{*}$ Silviu-Iulian Niculescu ${ }^{* *}$ \\ * Dept. Mathematics, University "Politehnica” of Bucharest, \\ Romania. E-mail: \\ constantin.morarescu@math.pub.ro \\ ** Laboratoire des Signaux et Systèmes (L2S), \\ CNRS-Supélec, Gif-sur-Yvette, France. E-mail: \\ Silviu.Niculescullss.supelec.fr.
}

\begin{abstract}
In this paper, we focus on the stability of some low-order distributed delay models describing cell-to-cell spread mechanisms in some well-mixed configuration. The stability analysis will be performed in the delay-parameter space defined by the mean delay and by the corresponding gap. We will explicitly compute the crossing frequency set, and the stability crossing curves by using a frequency-domain approach. An illustrative example encountered in HIV-1 infection will complete the presentation. Copyright (c) IFAC 2007
\end{abstract}

Keywords: distributed delay; biology; stability; crossing curves.

\section{INTRODUCTION}

Various biology model representations include a particular common element used to describe a reaction chain (distributed character, see, e.g.

In the sequel, we will focus on some particular class of dynamical models describing cell-to-cell spread mechanisms. Such systems are generally encountered in HIV-1 modeling as mentioned by

The remaining paper is organized as follows: the problem formulation and some comments on the system modeling can be found in Section 2. Section 3 includes the characterization of the stability regions in the delay-parameter set defined by the mean delay value and by the corresponding gap. An illustrative example corresponding to a HIV-1 model is presented in Section 4. Some concluding remarks end the paper. The notations are standard and explained when used for the first time.

\section{PROBLEM FORMULATION, AND SYSTEM MODEL}

In the sequel, we study a two-dimensional model of cell-to-cell spread mechanism, assuming that infection is spread directly from infected cells to healthy cells and neglecting the effects of free virus. The intracellular incubation period is given by a probability distribution and the model is given by two differential equations with distributed delay. More precisely, we consider the system

$$
\left\{\begin{array}{l}
\frac{\mathrm{d} C}{\mathrm{~d} t}=r_{C} C(t)\left(1-\frac{C(t)+I(t)}{C_{m}}\right)-k_{I} C(t) I(t) \\
\frac{\mathrm{d} I}{\mathrm{~d} t}=k_{I}^{\prime} \int_{-\infty}^{t} C(\theta) I(\theta) g(t-\theta) \mathrm{d} \theta-d_{I} I(t)
\end{array}\right.
$$

where $C(I)$ represents the concentration of healthy (infected) cells, $r_{C}$ is the reproductive rate of healthy cells, $C_{m}$ is the effective carrying capacity of the system, $k_{I}$ represents the infection of healthy cells by the infected cells in a well-mixed system, $k_{I}^{\prime} / k_{I}$ is 
the fraction of cells surviving the incubation period, $d_{I}$ is the death rate of infected cells, $g$ is a probability distribution that defines the infectious process history. Explicitly, we assume that the cells which are productively infectious at time $t$ were infected $\theta$ time ago, where $\theta$ is distributed according to the probability density $g$. Such a model is also encountered in the HIV-1 infection mechanisms as pointed out by

The initial conditions of the system (1) are defined as follows

$C(t)=\phi(t) \geq 0, \quad I(t)=\psi(t) \geq 0, \quad t \in(-\infty, 0]$, where $\phi$ and $\psi$ are continuous function on $(-\infty, 0]$.

The probability density $g$ that appears in system (1) is often replaced by some Dirac densities $g(\theta)=\delta(\theta)$, $g(\theta)=\delta(\theta-\tau)$ where $\tau$ is a constant or by a gamma distributed kernel

$$
g(\theta):=\frac{\alpha^{n+1} \theta^{n}}{n !} \mathrm{e}^{-\alpha \theta}
$$

where $\alpha>0$ and $n$ is a positive integer (see, for instance,

When $g(\theta)=\delta(\theta)$ one obtains the following ordinary differential equations (ODE):

$$
\left\{\begin{array}{l}
\frac{\mathrm{d} C}{\mathrm{~d} t}=r_{C} C(t)\left(1-\frac{C(t)+I(t)}{C_{m}}\right)-k_{I} C(t) I(t), \\
\frac{\mathrm{d} I}{\mathrm{~d} t}=k_{I}^{\prime} C(t) I(t)-d_{I} I(t) .
\end{array}\right.
$$

The initial conditions are

$$
C(0)=C_{0} \geq 0, \quad I(0)=I_{0} \geq 0,
$$

where $C_{0}$ and $I_{0}$ are constant.

When $g(\theta)=\delta(\theta-\tau)$ where $\tau$ is a constant, the system (1) becomes the following delay differential equations (DDE) with a discrete delay:

$$
\left\{\begin{array}{l}
\frac{\mathrm{d} C}{\mathrm{~d} t}=r_{C} C(t)\left(1-\frac{C(t)+I(t)}{C_{m}}\right)-k_{I} C(t) I(t), \\
\frac{\mathrm{d} I}{\mathrm{~d} t}=k_{I}^{\prime} C(t-\tau) I(t-\tau)-d_{I} I(t) .
\end{array}\right.
$$

The initial conditions are

$C(t)=\phi(t) \geq 0, \quad I(t)=\psi(t) \geq 0, \quad t \in[-\tau, 0]$,

where $\phi$ and $\psi$ are continuous function on $[-\tau, 0]$.

Finally, when

$$
g(\theta):=\frac{\alpha^{n+1} \theta^{n}}{n !} \mathrm{e}^{-\alpha \theta},
$$

we get a DDE with distributed delay. According to

In general, there is a short intracellular "eclipse phase" (often referred as "latency", see also the infection with HIV-1). During this period the cell is infected but has not yet begun producing virus.

The system (1) has three equilibrium points: the trivial equilibrium $(0,0)$, the healthy equilibrium $\left(C_{m}, 0\right)$ and the infected equilibrium $(\bar{C}, \bar{I})$, where

$$
\bar{C}=\frac{d_{I}}{k_{I}^{\prime}}, \quad \bar{I}=\frac{r_{C}\left(k_{I}^{\prime} C_{m}-d_{I}\right)}{k_{I}^{\prime}\left(r_{C}+k_{I} C_{m}\right)}
$$

if $k_{I}^{\prime}>d_{I} / C_{m}$. Note that the equilibrium points of the model do not depend on the choice of the probability density $g$. The interesting problem concerns the stability of the infected equilibrium point $(\bar{C}, \bar{I})$. Setting $C=\bar{C}+x, I=\bar{I}+y$ where $x$ and $y$ are small, and linearizing, one obtains the characteristic equation:

$$
\Delta(\lambda)=\lambda^{2}+p \lambda+r+(s \lambda+q) G(\lambda),
$$

where the coefficients above are given by:

$$
\begin{gathered}
p=\frac{d_{I}\left(k_{I}^{\prime} C_{m}+r_{C}\right)}{k_{I}^{\prime} C_{m}}, q=\frac{r_{C} d_{I}\left(k_{I}^{\prime} C_{m}-2 d_{I}\right)}{k_{I}^{\prime} C_{m}}, \\
r=\frac{r_{C} d_{I}^{2}}{k_{I}^{\prime} C_{m}}, s=-d_{I},
\end{gathered}
$$

and $G(\lambda)$ is the Laplace transform of $g(t)$. Next, replacing $g$ with the gamma distribution with a gap defined by (??) we get the following particular characteristic function:

$\Delta(\lambda ; T, \tau):=\left(\lambda^{2}+p \lambda+r\right)(1+\lambda T)^{n}+(s \lambda+q) \mathrm{e}^{-\lambda \tau}$.

\section{STABILITY CHARACTERIZATION IN THE DELAY PARAMETER SET}

In the sequel, we derive the stability regions of (5) in the delay parameter space $(T, \tau)$. In this sense, we will start by discussing a more general parameterdependent characteristic equation described by the following quasipolynomial:

$$
\Delta(\lambda ; T, \tau)=P(\lambda)(1+\lambda T)^{n}+Q(\lambda) \mathrm{e}^{-\lambda \tau}=0 .
$$

where $P$ and $Q$ are polynomials with real coefficients such that $\operatorname{deg}(Q)<\operatorname{deg}(P)$ (see, e.g.

Assumption I. $P(0)+Q(0) \neq 0$;

Assumption II. $P(\lambda)$ and $Q(\lambda)$ do not have common zeros;

Assumption III. If $P(\lambda)=\alpha, Q(\lambda)=\beta$, where $\alpha, \beta$ are real constants, then $|\alpha| \neq|\beta|$;

Assumption IV. $P(0) \neq 0,|P(0)| \neq|Q(0)|$;

Assumption V. $P^{\prime}(j \omega) \neq 0$ whenever $P(j \omega)=0$.

It is easy to see that in our case study, i.e. characteristic function $\Delta(\lambda ; T, \tau)$ given by (5), the Assumptions $\mathrm{I}$ and $\mathrm{V}$ are automatically satisfied, and the other reduces to some simple comparison of $P(0)=p$ with $Q(0)=q$. Indeed, Assumption I is equivalent to $k_{I}^{\prime} C_{m} \neq d_{I}$ which is automatically satisfied since, by hypothesis, we focus on the stability around a particular equilibrium, for which $k_{I}^{\prime}>d_{I} / C_{m}$.

Using the methodology proposed by

\subsection{Frequency crossing set computation}

Another useful concept is the frequency crossing set $\Omega$, which is defined as the collection of all $\omega>0$ 
such that there exists a delay-parameter pair $(T, \tau)$ such that $\Delta(j \omega ; T, \tau)=0$. In other words, as the parameters $T$ and $\tau$ vary, the characteristic roots may cross the imaginary axis at $j \omega$ if and only if $\omega \in \Omega$. Without any loss of generality, we can consider that $\Omega \subset \mathbb{R}_{+}$.

In the context of equation (6), the characterization of the frequency crossing set is given by the following:

Proposition 1. Given any $\omega>0, \omega \in \Omega$ if and only if it satisfies

$$
0<|P(j \omega)| \leq|Q(j \omega)|
$$

Consider now in more detail the inequality (7). First at all, it is easy to see that $\Omega$ is bounded, that is there exists a real positive number $M>0$, such that for all $\omega \in \Omega, \omega<M$. Indeed, since $\operatorname{deg}(P)>\operatorname{deg}(Q)$, then $\lim _{\omega \rightarrow \infty}|Q(j \omega)| /|P(j \omega)|=0$, and thus there exists some high-frequency $\omega_{h}>0$ such that $|P(j \omega)|>|Q(j \omega)|$, for all $\omega \geq \omega_{h}$, and thus for all $\omega>\omega_{h}$, (7) cannot be satisfied.

Next, $\Omega$ consists of a finite set of intervals of finite length. Indeed, it is easy to see that there are only a finite number of solutions of each of the following two equations

$$
P(j \omega)=0,
$$

and

$$
|P(j \omega)|=|Q(j \omega)|,
$$

because $P$ and $Q$ are both co-prime polynomials with $P(0)+Q(0) \neq 0$. Therefore, $\Omega$, which is the collection of $\omega$ satisfying (7), consists of a finite number of intervals. Denote these intervals as $\Omega_{1}, \Omega_{2}, \ldots, \Omega_{N}$. Then $\Omega=\bigcup_{k=1}^{N} \Omega_{k}$.

Without any loss of generality, we may order these intervals from left to right, i.e., for any $\omega_{1} \in \Omega_{k_{1}}$, $\omega_{2} \in \Omega_{k_{2}}, k_{1}<k_{2}$, we have $\omega_{1}<\omega_{2}$.

\subsection{Stability crossing curves characterization}

Using the results and the notations above, we arrive to the following characterization of the stability crossing curves:

Proposition 2. The set $\mathcal{T}$ consists of all the pairs $(T, \tau)$ given by

$$
\begin{gathered}
T=\frac{1}{\omega}\left(\left|\frac{Q(j \omega)}{P(j \omega)}\right|^{2 / n}-1\right)^{1 / 2} \\
\tau=\tau_{m}=\frac{1}{\omega}(\angle Q(j \omega)-\angle P(j \omega)-n \arctan (\omega T) \\
+\pi+2 m \pi) \\
m=0, \pm 1, \pm 2, \ldots .
\end{gathered}
$$

where $\omega \in \Omega$ represents a crossing frequency.
We will not restrict $\angle Q(j \omega)$ and $\angle P(j \omega)$ to a $2 \pi$ range. Rather, we allow them to vary continuously within each interval $\Omega_{k}$. Thus, for each fixed $m$, (10) and (11) represent a continuous curve. We denote such a curve as $\mathcal{T}_{m}^{k}$. Therefore, corresponding to a given interval $\Omega_{k}$, we have an infinite number of continuous stability crossing curves $\mathcal{T}_{m}^{k}, m=0, \pm 1, \pm 2, \ldots$. It should be noted that, for some $m$, part or the entire curve may be outside of the range $\mathbb{R}_{+}^{2}$, and therefore, may not be physically meaningful. The collection of all the points in $\mathcal{T}$ corresponding to $\Omega_{k}$ may be expressed as

$$
\mathcal{T}^{k}=\bigcup_{m=-\infty}^{+\infty}\left(\mathcal{T}_{m}^{k} \bigcap \mathbb{R}_{+}^{2}\right)
$$

Obviously, $\mathcal{T}=\bigcup_{k=1}^{N} \mathcal{T}^{k}$.

\subsection{Classification of stability crossing curves}

Let the left and right end points of interval $\Omega_{k}$ be denoted as $\omega_{k}^{\ell}$ and $\omega_{k}^{r}$, respectively. Due to Assumptions IV and $\mathrm{V}$, it is not difficult to see that each end point $\omega_{k}^{\ell}$ or $\omega_{k}^{r}$ must belong to one, and only one, of the following three types:

Type 1. It satisfies the equation (9).

Type 2. It satisfies the equation (8).

Type 3. It equals 0 .

Denote an end point as $\omega_{0}$, which may be either a left end or a right end of an interval $\Omega_{k}$. Then the corresponding points in $\mathcal{T}_{m}^{k}$ may be described as follows.

If $\omega_{0}$ is of type 1 , then $T=0$. In other words, $\mathcal{T}_{m}^{k}$ intersects the $\tau$-axis at $\omega=\omega_{0}$.

If $\omega_{0}$ is of type 2 , then as $\omega \rightarrow \omega_{0}, T \rightarrow \infty$ and $\tau \rightarrow$

$\frac{1}{\omega_{0}}\left(\angle Q\left(j \omega_{0}\right)-\lim _{\omega \rightarrow \omega_{0}} \angle P(j \omega)-\frac{n \pi}{2}+\pi+m 2 \pi\right)$.

Obviously,

$$
\lim _{\omega \rightarrow \omega_{0}} \angle P(j \omega)=\angle\left[\frac{d}{d \omega} P(j \omega)\right]_{\omega \rightarrow \omega_{0}}
$$

if $\omega_{0}$ is the left end point $\omega_{k}^{\ell}$ of $\Omega_{k}$, and

$$
\lim _{\omega \rightarrow \omega_{0}} \angle P(j \omega)=\angle\left[\frac{d}{d \omega} P(j \omega)\right]_{\omega \rightarrow \omega_{0}}+\pi
$$

if $\omega_{0}$ is the right end point $\omega_{k}^{r}$ of $\Omega_{k}$. In other words, $\mathcal{T}_{m}^{k}$ approaches a horizontal line. Obviously, only $\omega_{1}^{\ell}$ may be of type 3 . Due to non-singularity assumption IV, if $\omega_{1}^{\ell}=0$, we must have $0<|P(0)|<|Q(0)|$. In this case, as $\omega \rightarrow 0$, both $T$ and $\tau$ approach $\infty$. In fact, $(T, \tau)$ approaches a straight line with slope

$\tau / T \rightarrow \frac{(\angle Q(0)-\angle P(0)-n \arctan \alpha+\pi+m 2 \pi)}{\alpha}$, 
where

$$
\alpha=\left(\left|\frac{Q(0)}{P(0)}\right|^{2 / n}-1\right)^{1 / 2}
$$

$2 \times 10^{6} / \mathrm{mL}, k_{I}=2 \times 10^{-6} / \mathrm{mL} /$ day,$k_{I}^{\prime}=1.5 \times$ $10^{-6}, d_{I}=0.3 /$ day,$r_{C}=0.68 /$ day $($ see

We say an interval $\Omega_{k}$ is of type $\ell r$ if its left end is of type $\ell$ and its right end is of type $r$. We may accordingly divide these intervals into the following 6 types.

Type 11. In this case, $\mathcal{T}_{m}^{k}$ starts at a point on the $\tau$ axis, and ends at another point on the $\tau$-axis.

\section{REFERENCES}

Baker, C.T.H., G.A. Bocharov and F.A. Rihan (1999). A report on the use of delay differential equations in numerical modelling in the biosciences. Numerical Analysis Report No. 343, Manchester Centre for Computational Mathematics, Manchester.

Type 12. In this case, $\mathcal{T}_{m}^{k}$ starts at a point on theCulshaw, R.V., S. Ruan and G. Webb (2003). A mathe$\tau$-axis, and the other end approaches $\infty$ along a horizontal line.

Type 21. This is the reverse of type $12 . \mathcal{T}_{m}^{k}$ starts at matical model of cell-to-cell spread of HIV-1 that includes a time delay. J. Math. Biology, vol. 46, pp. 425-444.

$\infty$ along a horizontal line, and ends at the $\tau$-axis. Cushing, J.M. (1981). Volterra integrodifferential equations

Type 22. In this case, both ends of $\mathcal{T}_{m}^{k}$ approach horizontal lines.

Type 31. In this case, $\mathcal{T}_{m}^{k}$ begins at $\infty$ with an in population dynamics. in Mathematics of Biology (M. IANNALLI, EDS. Ligouri Editore: Naples), 81148.

asymptote of slope expressed in (15). The other endGopalsamy, K. (1992). Stability and oscillations in deis on the $\tau$-axis.

Type 32. In this case, $\mathcal{T}_{m}^{k}$ again begins at $\infty$ with an lay differential equations of population dynamics, Kluwer Academic, Math. Its Appl., 74.

asymptote of slope expressed in (15). The other endGourley, S.A. and Y. Kuang (1994). A delay reactionapproaches $\infty$ along a horizontal line.

Several examples covering the cases mentioned above can be found in diffusion lodel of the spread of bacteriophage infection. Internal Note Arizona State University, 1994.

Hoppensteadt, F.C. and C.S. Peskin (1992). Mathematics in Medicine and Life Sciences, Springer-Verlag: New York, TAM, 10.

\subsection{Crossing direction characterization}

Next, introduce the following notations:

$$
\begin{aligned}
R_{0} & =\operatorname{Re}\left(\frac{j}{\lambda} \frac{\partial \Delta(\lambda, T, \tau)}{\partial \lambda}\right)_{\lambda=j \omega}, \\
I_{0} & =\operatorname{Im}\left(\frac{j}{\lambda} \frac{\partial \Delta(\lambda, T, \tau)}{\partial \lambda}\right)_{\lambda=j \omega}, \\
R_{1} & =\operatorname{Re}\left(\frac{1}{\lambda} \frac{\partial \Delta(\lambda, T, \tau)}{\partial T}\right)_{\lambda=j \omega}, \\
I_{1} & =\operatorname{Im}\left(\frac{1}{\lambda} \frac{\partial \Delta(\lambda, T, \tau)}{\partial T}\right)_{\lambda=j \omega} \\
R_{2} & =\operatorname{Re}\left(\frac{1}{\lambda} \frac{\partial \Delta(\lambda, T, \tau)}{\partial \tau}\right)_{\lambda=j \omega}, \\
I_{2} & =\operatorname{Im}\left(\frac{1}{\lambda} \frac{\partial \Delta(\lambda, T, \tau)}{\partial \tau}\right)_{\lambda=j \omega},
\end{aligned}
$$

the crossing direction is given by the following:

Proposition 3. Let $\omega \in \Omega$ and $(T, \tau) \in \mathcal{T}$ such that $j \omega$ is a simple solution of (6) and

$$
\Delta\left(j \omega^{\prime} ; T, \tau\right) \neq 0, \forall \omega^{\prime}>0, \omega^{\prime} \neq \omega
$$

(i.e. $(T, \tau)$ is not an intersection point of two curves or different sections of a single curve of $\mathcal{T}$ ). Then a pair of solutions of (6) cross the imaginary axis to the right, through $\lambda= \pm j \omega$ if

$$
R_{2} I_{1}-R_{1} I_{2}>0 .
$$

The crossing is to the left if the inequality is reversed.

\section{ILLUSTRATIVE EXAMPLE}

Consider now the case of a cell-to-cell spread model representing HIV infection mechanisms with $C_{m}=$
Kuang, Y. (1993). Delay differential equations with applications in population dynamics, Academic Press: Boston.

Leonard, R., Zagury, D., Desports, I., Bernard, J., Zagury, J.-F. and R.C. Gallo (1988). Cytopathic effect of human immunodeficiency virus in T4 cells is linked to the last stage of virus infection. Proc. Natl. Acad. Sci. USA, vol. 85, pp. 3570-3574.

MacDonald, N. (1989). Biological delay systems: linear stability theory. (Cambridge University Press, Cambridge).

Mittler, J.E., B. Sulzer, A.U. Neumann and A.S. Perelson (1998). Influence of delayed viral production on viral dynamics in HIV-1 infected patients. Math. Biosciences vol. 152, pp. 143-163.

Morărescu, C.I. (2006). Qualitative analysis of distributed delay systems: Methodology and algorithms, Ph.D. thesis, University of Bucharest/Université de Technologie de Compiègne, September.

Murray, J.D. (1993). Mathematical Biology, Springer: Berlin (2nd Edition), BioMath., 18.

Niculescu, S.-I., W. Michiels, I.C. Morărescu, and K. Gu (2007). Geometric ideas in the stability analysis of delay systems in biosciences. in Biology and control theory: current challenges, LNCIS, 357, SpringerVerlag: Berlin, 217-259.

Spouge, J.L., R.I. Shrager, and D.S. Dimitrov (1996). HIV1 infection kinetics in tissue cultures, Math. Biosciences, vol. 138, pp. 1-22.

Vielle, B. and G. Chauvet. (1998). Delay equation analysis of human respiratory stability, Math. Biosciences, vol. 152, pp. 105-122. 